\title{
The Ensuring of the Economic Security of Industrial Enterprises in the Context of Forming a Flexible Management Model: Prerequisites and Tools
}

\author{
Larysa Liubokhynets *[0000-0003-0958-130X], Yevhenii Rudnichenko [0000-0002-9407-2026], \\ Nataliia Havlovska ${ }^{\text {[0000-0003-1084-2853] }}$
}

Khmelnitsky National University, Khmelnitsky, Ukraine

*lubohinets@ukr.net

\begin{abstract}
In the article the prerequisites for building a model of flexible enterprise management in order to ensure its economic security examines. It is determined that directive management methods, although they ensure the operative execution of tasks, in many cases do not contain a sufficient level of flexibility, and this does not allow the economic security system to function effectively. To build a model of flexible management it is propose to use intelligent data processing systems based on the construction and use of neural networks. The preconditions for the formation of the model and the main factors influencing on it are determined. The main groups of stakeholders that interested in developing a model of flexible management to ensure the economic security of the enterprise presented; their goals and expected results defined. It is substantiated that a probabilistic neural network is expedient to build in the Matlab environment, which ensures the efficiency and relevance of the obtained results.
\end{abstract}

Keywords: economic security, flexible management, neural network, model, tools, stakeholders.

\section{INTRODUCTION}

The economic security of industrial enterprises largely depends on the flexibility of management processes. The obtained results of assessing the flexibility of the management system [1] of industrial enterprises [2] indicate the need for significant improvements in management processes, and in some cases their new architectonics. Standardized, mostly directive management methods, although they ensure the prompt execution of tasks, but in many cases do not contain a sufficient level of flexibility. A special place in the formation of a system of flexible management is occupy the management decisions that directly implement the reactions of enterprise management and ensure the functioning of such a system that based on information about the internal and external environment of business entities [3, 4].

\section{METHODS}

To ensure the economic security of enterprises, management needs and improve the decision-making system, it is expedient to use intelligent data processing systems based on the construction and use of neural networks.

The choice of a probabilistic neural network for building a system of flexible management and ensuring the economic security can be justified as follows. Since in the practice we have a list of $\mathrm{N}$ features that have be matched to a certain condition of the economic security system, which is characterized by these features, we should build a reflection of this list in the condition. In fact, we are talking about some function specified in the list of conditions. This function should as accurately as possible reflect an arbitrary list of features in the corresponding condition of the economic security system. However, we must take into account that such a function will depend on the $N$ variables. In addition, although each feature (factor influencing the threat) in our case has a finite set of potential values, the desired function cannot be considered as given on a discrete (and, in fact, finite) lattice set of its arguments [5-7]. If it were so, then it would be possible to compile a kind of correspondence table between all the variants of the features lists and conditions, after which the task of classifying the condition according to the list of $N$ 
features, would solved in a trivial way. However, each feature (after normalization) can have any value in the range from 0 to 1 , because it will be determined on the basis of aggregation (averaging) of expert opinions. Therefore, the corresponding reflection of $N$ variables (list of features) to the condition will not be a discrete function.

Therefore, we need to construct a statistical classifier of $N$ features. To implement the statistical classifier, we have the following options:

1) regression construction (linear $[8,9]$ or nonlinear);

2) building a decision tree;

3) construction and training of a two-layer perceptron;

4) construction of a neural network based on the radial basis functions (probabilistic neural network, general regression neural network, radial-basic neural network, exact radial-basic neural network);

5) Bayes' classifiers;

6) SVM type classifiers;

7) neural networks of deep learning.

The best approach within the research is to classify (diagnose) based on a probabilistic neural network, which is the most accurate of all neural networks using radial basis functions.

\section{RESULTS}

To form a model of flexible enterprise management based on the neural network, it is necessary to determine the prerequisites for its formation and the factors of influence, which include the following. Unpredictable events and threats of global scale (pandemics, wars, natural disasters) in today's conditions are becoming a reality for most enterprises, regardless of country, industry or scale of activity. The global COVID 19 pandemic and military action at the national level have an extremely negative impact on business activities by generating appropriate information waves to which management is forced to respond. Transformations of target markets are constantly taking place, which in most cases worsen the situation for producers and the industrial sector as a whole. Information about the state of markets and changes that significantly affected on demand is extremely useful and relevant for making strategic decisions and adjusting the overall strategy of enterprise development.

Aspects of the influence of the domestic institutional environment on the activities of enterprises [10-12] allow us to state its high turbulence and resistance to progressive changes, which causes threats and dangers to which the enterprise is extremely difficult to respond. The instability of the institutional environment is associated with possible illegal actions of third parties (raiding, takeovers and other actions), the negative influence of regulatory authorities (primarily fiscal), and the ambiguous influence of local governments.

However, not only the institutional environment, but also the competitive environment generates a number of threats related to: competition for markets; struggle for resources and logistics flows; lobbying interests at the macro- and medium levels; struggle for government order and budget funding.

To the above conditions of functioning and development of the enterprise's environment are "connected" the internal features of each enterprise. From the standpoint of economic security, such threats must be level by an effective system of economic security, the operation of which based on information and management decisions. In addition, if information is the "blood" of the system, then management decisions - its "nerve impulses". The inertia of one of the two components in the management system of the enterprise leads to the emergence of time "lags" in response to external stimuli and does not allow the economic security system to work with a sufficient level of efficiency.

At the same time, diverting the attention of managers to minor changes in the environment of the enterprise operation, or to those that are systematically repeat, will not bring tangible benefits to ensure economic security. Hence, there are certain requirements, both for information and for analytical support of management processes, and for the tools and instruments that used in modeling changes in the management system. In this case, the priority in this context will be compliance with the principles of management flexibility. The main requirements for information and analytical support of management processes from the standpoint of economic security ensuring include: efficiency; certainty; precision; managerial value; scale.

Such requirements are mostly determined by the basic principles of functioning of the mechanism of management of the economic security system of the enterprise, as indicated in [13] are: systematic; complexity; adaptability; interactivity; legality; alternative; consistency of management goals; cycle; interdisciplinary approach; the principle of individualization; the principle of economic feasibility. In general, agreeing with the author on the above given principles, it should be noted the lack of the principle of flexibility of the mechanism's response to external and internal stimuli. Accordingly, the task is to form a flexible management system based on the use of modern management tools and intelligent data processing systems with the subsequent formation of an appropriate model. The basic conditions for developing a model of flexible management to ensure the economic security of the enterprise shown in Figure 1. 


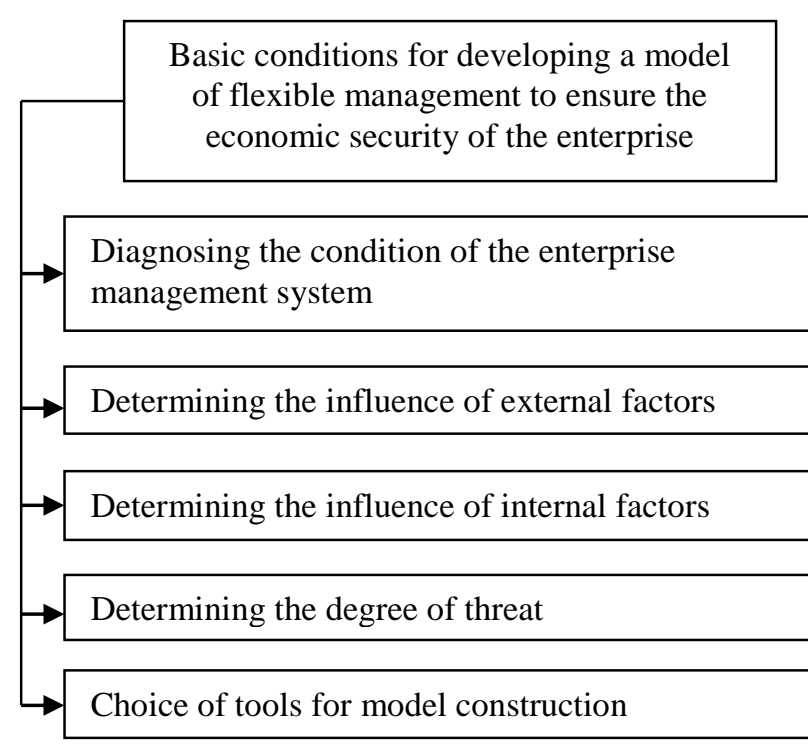

Figure 1 Basic conditions for the development of flexible management models to ensure the economic security of the enterprise

The initial condition for building a model of flexible management to ensure the economic security of the enterprise is to diagnose the condition of the enterprise management system as a whole. Such diagnosis allows identifying the "bottlenecks" of the management system and the actual reactions to external influences. The internal resistance of the management system to innovations also identified. Such aspects have be taken into account when building the model. In addition, it is necessary to determine the influence of external and internal factors that shape the demand for adaptability and flexibility of the management system, as well as determine its complexity from the standpoint of the architectonics of the management structure and the establishment of internal organizational relations.

It is clear that not all factors will have a negative impact and generate threats to the enterprise activity. Therefore, for the system of economic security of the enterprise, as well as to improve the management system, it is necessary to determine the degree of threat, from the critical level to the level of ignorance.

Taking into account the conditions of model development is a mandatory component of modeling, but it is necessary to identify the main stakeholders who are interested in building such a model and their motives for spending resources (Table 1).

The main groups of stakeholders include management, owners, employees, economic security service of the enterprise. Each group of stakeholders has its own goals and expected results from the implementation of the model in the practice of enterprises.
Table 1. The main groups of stakeholders that interested in developing a model of flexible management to ensure the economic security of the enterprise

\begin{tabular}{|c|c|c|}
\hline $\begin{array}{l}\text { A group of } \\
\text { stakeholders }\end{array}$ & Objectives & Expected result \\
\hline Management & $\begin{array}{lr}\text { Efficiency } & \text { and } \\
\text { adequacy } & \text { of } \\
\text { management } \\
\text { decisions to existing } \\
\text { realities } \\
\text { challenges }\end{array}$ & $\begin{array}{l}\text { Improving } \\
\text { management } \\
\text { efficiency in } \\
\text { general and } \\
\text { personalized } \\
\text { income }\end{array}$ \\
\hline Owners & $\begin{array}{l}\text { Increasing the } \\
\text { transparency of } \\
\text { management } \\
\text { processes and } \\
\text { adaptation of the } \\
\text { enterprise to market } \\
\text { conditions, } \\
\text { increasing } \\
\text { competitiveness }\end{array}$ & $\begin{array}{l}\text { The growth of the } \\
\text { market value of } \\
\text { the company and } \\
\text { the welfare of the } \\
\text { owners }\end{array}$ \\
\hline Employees & $\begin{array}{l}\text { Determining } \\
\text { personal } \\
\text { contribution to } \\
\text { achieving the overall } \\
\text { strategic goals of the } \\
\text { company, the } \\
\text { distribution of } \\
\text { responsibilities }\end{array}$ & $\begin{array}{l}\text { The identification } \\
\text { of employee value } \\
\text { for the company } \\
\text { and the formation } \\
\text { of an effective } \\
\text { system of } \\
\text { motivation }\end{array}$ \\
\hline $\begin{array}{l}\text { Enterprise } \\
\text { economic } \\
\text { security } \\
\text { department }\end{array}$ & $\begin{array}{l}\text { The fastest response } \\
\text { to external negative } \\
\text { influences and } \\
\text { internal threats, } \\
\text { minimizing risks }\end{array}$ & $\begin{array}{l}\text { Increasing the } \\
\text { level of business } \\
\text { security }\end{array}$ \\
\hline
\end{tabular}

For enterprise management, the main goal is the efficiency and adequacy of management decisions to existing realities and challenges. The main expected result is an increase in management efficiency in general and personalized income in the form of bonuses for achieving the target performance of the enterprise, which is a common practice in most companies around the world.

Owners are interested in increasing the transparency of management processes and adapting the enterprise to the market conditions, which increases its competitiveness. Accordingly, if these goals achieved, there is an increase in the market value of the company and the welfare of the owners.

Employees of the enterprise (the majority) are interested in determining the personal contribution to achieving the overall strategic goals of the company, as well as in a clear division of responsibilities, which in result leads to identifying employee value for the company and forming an effective system of motivation.

The economic security service of the enterprise, as one of the subjects of management, is interested in the fastest possible reaction to external negative influences and internal threats. Flexible management also involves minimizing the risks that arise due to unreasonable or too time-consuming management decisions. As a result, it increases the level of business security. 
The motives for developing and implementing a model of flexible management differ slightly in different groups of stakeholders, but the common feature for them is the individual interest in implementing such a model in practice and a positive impact on the management processes of the enterprise as a whole.

For formation an effective model, it is necessary to provide information and analytical support, because without the necessary information and its detailed analysis, the model will not work with the expected level of efficiency. It has to be noted that a standardized approach with the analysis and collection of information on the external and internal environment is useful, but does not give an idea about specifics of the functioning and formation of management processes by subjects of managerial influence and meeting their needs from the standpoint of influencing on the economic security.

Accordingly, only after a thorough preparation and diagnosis of the state of information and analytical support of the management system of the enterprise, it is advisable to proceed to the stage of choosing the tools to build a model.

Note that under the management tools of the enterprise we mean such levers of influence, the implementation of which involves flexible management. Flexible management, in particular, also aims at the gradual implementation of management decisions, without "abrupt" transformations or restructuring that could "destroy" the system as a whole. That is, flexible management is equivalent to the implementation of a number of certain adjustments. This is, firstly, necessary in order to see the reaction of the environment, to see its "sensitivity" to the actions of management (senior management) of the enterprise.

What management tools of the enterprise should we consider? First of all, it is necessary to accept and remember the principle of minimal (insignificant) changes when using these tools. For example, if one of the most influential tools is the regulation of wages, then, of course, such regulation cannot have too long intervals. Indeed, wages cannot be raised rapidly, even if such increases motivated by inflation. Especially if the company has the opportunity and desire to stimulate its employees. Thus, the regulation of wages, changes in bonuses, as well as penalization (for, for example, late or non-fulfillment of the production plan) for a specific period should be "soft" and gradual. Gradualism is also provided in the regulation (reduction or increase) of production, reduction or increase of staff, changes in the structure of the enterprise, changes in branding (rebranding), etc. This gradualness is intended primarily to study the reaction of the external economic environment. Once this response will be more or less known and predictable, the implementation of management tools may become more stringent.
Flexible management involves periodic "scanning" of the economic environment (both external and internal), which directly or indirectly effects on the economic activity of the enterprise, the sustainability and stability of its development. According to the results of the scan, certain management decisions will made to prevent the deterioration of the economic condition of the enterprise. In other words, flexible enterprise management serves to ensure a sufficient level of economic security. A sufficient level of economic security is manifest in the ability of the economic security system to prevent and respond to threats according to the dynamics of economic processes development, which allows ensuring the functioning and strategic development of the enterprise and maximizing its market value in the medium term.

However, it is impossible to specify the duration of this medium-term perspective. The shortest period for which it is possible to "scan" the economic environment appropriate to the interests of the enterprise, can be measure in weeks. On the other hand, the response of the economic environment may be so slow that the steps of flexible management may be longer in time. It can be a month or even a quarter, but no longer. It should be note that the amount of adjustments might already be greater than for shorter periods of "scanning" and making a management decision. Thus, flexible management with a longer waiting interval involves more "abrupt" transformations and adjustments. At the same time, there is no reason to say that such an amplitude of flexible management is more typical for large enterprises. The fact is that the amplitude of flexible management depends, in particular, on inflation, which, unfortunately, has recently intensified in our country. This is also one of the threats to the economic security of the enterprise, which is very difficult to counter. Moreover, there are a generalized number of other threats, the lack of counteraction or prejudice of which can have devastating consequences for the economic condition of the enterprise.

Inflationary processes pose, of course, a serious threat to the economy of any enterprise. However, deflationary processes can be destructive. In general, exchange rate fluctuations are one of the most influential threats to the economic security of the enterprise. Among the groups of other potential threats are weak market predictability (demand), excitement (caused by unpredictable changes in legislation, new laws or even just their projects, unreasonable increase in the minimum wage), loss of competitiveness, deterioration of resource supply, aggravation of interpersonal relationships on the enterprise.

\section{CONCLUSIONS}

The system of flexible management of the enterprise to ensure a sufficient level of its economic security should built based on statistical classifiers [14]. Firstly, 
we need to identify a list of factors influencing the degree of threat. Denote by:

$$
Z=\left\{z_{i}\right\}_{i=1}^{N}
$$

The set of all such factors, where $z_{i}$ is the $i$-th factor or the $i$-th feature (according to the terminology of statistical classifiers), which negatively effects on the degree of threat (i.e., increases this degree), and $N$ is the total number of these features. For example, all factors of influence (signs) can be divide into external and internal.

In this example, we can see that set (1) may actually contain several dozen influencing factors. Based on them, it is necessary to determine the degree of threat. For further consideration, the set (1) is represent as a combination of subsets of external and internal factors:

$Z=\left\{z_{i}\right\}_{i=1}^{N}=Z_{\text {ext }} \cup Z_{\text {int }}=\left\{z_{i_{1}}^{(\text {ext })}\right\}_{i_{1}=1}^{N_{\text {ext }}} \cup\left\{z_{i_{2}}^{(\text {int })}\right\}_{i_{2}=1}^{N_{\text {int }}}$

Where $Z_{\text {ext }}$ and $Z_{\text {int }}$ are subsets of $N_{\text {ext }}$ external and $N_{\text {int }}$ internal factors, moreover $N=N_{\text {ext }}+N_{\text {int }}$.

Denote by:

$$
S=\left\{s_{k}\right\}_{k=1}^{M}
$$

The set of $M$ degrees of threat to the economic security of the enterprise, in which $s_{k}$ is the $k$-th degree (type or type) of threat. For example, the set (3) can be formed from four degrees of threat (in ascending order of potential negative consequences): weak, moderate, significant (strong), critical. Of course, such a division is conditional, and it can vary for companies in even one industry, not to mention companies in different industries. The larger the enterprise, the greater the degree of threat to be considered, because the difference between neighboring levels (for example, between weak and moderate) for a larger enterprise means a greater difference in potential losses from inaction in countering threats.

Thus, formally speaking, we are faced with the task of not just mapping the set of factors influencing $Z$ into the set of threat levels $S$, but the task of classifying the degree of threat based on the study of $N$ features in the set $Z$. In other words, we need to make a choice:

$$
s^{*} \in S=\left\{s_{k}\right\}_{k=1}^{M}
$$

Where $s^{*}$ is the most relevant threat level among all $M$ levels. This can be done using a statistical classifier such as a probabilistic neural network. A probabilistic neural network is a mathematical object that implements a three-step computational procedure. This procedure takes place on the input, radial and output layers of the neural network [15].
A probabilistic neural network is very easy to build in a Matlab environment. To do this, it is necessary to form an input matrix [16]:

$$
\mathbf{F}=\left[f_{i j}\right]_{N \times(m M)}
$$

where $m \in\{1,2,3, \ldots\}$, and the value of the element $f_{i j}$ is a numerical estimate (fractional or integer, as well as from an arbitrary, generally speaking, interval) of the sign $z_{i}$ in the state (class) $s_{k}$ for:

$$
k=j-M \cdot \psi\left(\frac{j-1}{M}\right)
$$

where the auxiliary function $\Psi(x)$ returns an integer part of the number $x$. Theoretically, the matrix (5) can have the size $N \times \mathrm{M}$, where $m=1$ and $k=j$ by formula (6). In this case, each sign in each state (degree of threat) will have only one assessment. This case is possible in the calculations, but its implementation requires very reliable estimates (all you need to provide $N \cdot M$ of such estimates).

This approach can significantly improve the management system of the enterprise and ensures the economic security of industrial enterprises by increasing the speed of response of the management system to the negative impact of the environment. The proposed approach requires further research in the context of testing and progress of developed approaches.

\section{REFERENCES}

[1] Abimbola, R. Iyanda Olufemi, D. Ninan and Damilola, J. Odejimi (2018), "Students Conversation Management System", International Journal of Education and Management Engineering, vol. 8, no. 4, pp. 1-9, DOI: $10.5815 /$ ijeme.2018.04.01

[2] Rudnichenko, Y. Liubokhynets, L. Havlovska, N. Illiashenko, O. and Avanesova, N. (2021), "Qualitative Justification of Strategic Management Decisions in Choosing Agile Management Methodologies", International Journal for Quality Research, vol. 15(1), pp. 209-224, DOI: $10.24874 / \mathrm{IJQR} 15.01-12$

[3] Havlovska, N. Rudnichenko, Ye. Babiy, I. Matiukh, S. Liubokhynets, L. and Lopatovskyi, V. (2020), "The strategy of choosing promising markets for the enterprise - subject of foreign economic activity on the basis of accessibility, safety and profitability criteria", Quality-Access to Success Journal, vol. 21(178), pp. 26-34.

[4] Rudnichenko, Y. Krymchak, L. Kudelskyi, V. Avanesova, N. Sokyrnyk, I. and Havlovska, N. (2020), "Minimization of risks of the enterprise foreign economic activity through improving the 
interaction management quality with potential partners", Quality-Access to Success Journal, vol. 21 (174), pp. 61-67.

[5] Kannaiya Raja, N. Saritha, R. Senthamaraiselvan, A. and Arulanandam, K. (2012), "QSWMCA Quality of Service in Wireless Mesh Networks by Configuration Arguments", IJCNIS, vol. 4, no. 6, pp. 1-9, DOI: 10.5815/ijcnis.2012.06.01

[6] Qi, Wang and Fenglian, Fu (2012), "Variational Iteration Method for Solving Differential Equations with Piecewise Constant Arguments", IJEM, vol. 2, no. 2, pp. 36-43, DOI: 10.5815/ijem.2012.02.06

[7] Qi, Wang and FengLian, Fu (2011), "Numerical Oscillations of Runge-Kutta Methods for Differential Equations with Piecewise Constant Arguments of Alternately Advanced and Retarded Type", International Journal of Intelligent Systems and Applications, vol. 3, no. 4, pp. 49-55, DOI: $10.5815 /$ ijisa.2011.04.07

[8] Korystin, Oleksandr and Svyrydiuk, Nataliia (2021), "Activities of Illegal Weapons Criminal Component of Hybrid Threats", Proceedings of the International Conference on Economics, Law and Education Research (ELER 2021), vol. 170, 22 March, pp. 86-91,
[9] Korystin, Oleksandr and Svyrydiuk, Nataliia (2020), "Methodological principles of risk assessment in law enforcement activity", Nauka $i$ Pravookhorona, vol. 3 (49), pp. 191-198, DOI: $10.36486 / \mathrm{np} .2020 .3(49) .19$

[10] Boland Lawrence, A. (1979), "Knowledge and the role of institutions in economic theory", Journal of Economic, vol. 13 (4), pp. 957-972.

[11] Comons, J. (1934), Institutional economics: Its place in political economy, New York, McMillan.

[12] Knight, J. (1992), Institutions and social conflict, New York, Cambridge University Press.

[13] Illiashenko, O.V. (2016), Mechanisms of the system of economic security of enterprise, monograph, Kharkiv.

[14] Dreyfus, G. (2005), Neural Networks: Methodology and Applications, Springer-Verlag Berlin Heidelberg.

[15] Haenni, R. Romeijn, J.-W. Wheeler, G. and Williamson, J. (2011), Probabilistic Logics and Probabilistic Networks, Springer Netherlands.

[16] Masters, T. (1993), Probabilistic Neural Networks, Practical Neural Network Recipies in $\mathrm{C}++$, Academic Press. 